INPLASY

PROTOCOL

To cite: Cavaleiro et al. Talent identification and development in male football? Systematic review updated (2017-2021). Inplasy protocol 202210074. doi:

10.37766/inplasy2022.1.0074

Received: 15 January 2022

Published: 15 January 2022

Corresponding author:

Vasco Cavaleiro

vasco.cavaleiro@gmail.com

Author Affiliation:

Universidade da Beira Interior

- Departamento de Ciências

do Desporto.

Support: NA.

Review Stage at time of this submission: Piloting of the study selection process.

\section{Talent identification and development in male football? Systematic review updated (2017-2021)}

Cavaleiro, V1; Travassos, B2; Mendes, R3; Sarmento, $\mathrm{H}^{4}$.

Review question / Objective: What are the constrain that have a direct influence in the process of talent identification and development in male football players?

Condition being studied: Constrains associated with talent identification and development, namely: (1) task constrains; (2) performers' constrains; (3) Environmental constrains.

Eligibility criteria: The publications included in the first search round met the following criteria: (1) articles written between 2017 and 2021 inclusive; (2) contained relevant data concerning talent identification and/or development; (3) were performed on male footballers; and (4) were written in the English language. Studies were excluded if they (1) included practitioners of other sports; (2) included females; and (3) did not contain any relevant data on talent development.

INPLASY registration number: This protocol was registered with the International Platform of Registered Systematic Review and Meta-Analysis Protocols (INPLASY) on 15 January 2022 and was last updated on 15 January 2022 (registration number INPLASY202210076).

Conflicts of interest:

None declared.

\section{INTRODUCTION}

Review question / Objective: What are the constrain that have a direct influence in the process of talent identification and development in male football players?

Condition being studied: Constrains associated with talent identification and development, namely: (1) task constrains; (2) performers' constrains; (3) Environmental constrains.

\section{METHODS}

Search strategy: A systematic review of Web of Science (all databases), Scopus and PubMed databases will be performed 
according to PRISMA (Preferred Reporting Items for Systematic Reviews and Metaanalyses) guidelines. The following keywords were used: "football" and "soccer". Each word was associated with the terms 'talent*', expert*, elite, "elite athlete", "talent identification", "talent development", "career transition" or "career progression". The selection was for the original articles in English containing relevant data about talent development/ identification on male footballers.

Participant or population: Male football players.

Intervention: Studies on talent identification and development related areas.

Comparator: The results will be compared with the following systematic review: Sarmento, H., Anguera, M. T., Pereira, A., \& Araújo, D. (2018). Talent identification and development in male football: a systematic review. Sports medicine, 48(4), 907-931.

\section{Study designs to be included: None.}

Eligibility criteria: The publications included in the first search round met the following criteria: (1) articles written between 2017 and 2021 inclusive; (2) contained relevant data concerning talent identification and/or development; (3) were performed on male footballers; and (4) were written in the English language. Studies were excluded if they (1) included practitioners of other sports; (2) included females; and (3) did not contain any relevant data on talent development.

Information sources: Web of Science (All databases), Scopus and PubMed databases.

Main outcome(s): The main findings of the sitematic review are divided into three major groups: task constraints, performance constraints and environmental constraints. Task constraints refer to the specificity and amount of football specific practice. Performer constraints include psychological factors, technical and tactical skills and anthropometric and physiological factors. Environmental constraints refer to the relative effect of age and socio-cultural influences.

\section{Additional outcome(s): NA.}

Data management: To be carried out according to PRISMA (Preferred Reporting Items for Systematic Reviews and MetaAnalyses) Guidelines for reviewing selected scientific articles.

Quality assessment / Risk of bias analysis: The overall methodological quality of the studies was assessed using the Critical Review Forms in Letts et al. (2007), or qualitative studies (counting 21 items), and by consulting Law et al. (1998) for quantitative studies (counting 16 items).

Strategy of data synthesis: As in previous systematic review of Sarmento et al (2018), a data extraction sheet (from Cochrane Consumers and Communication Review Group's data extraction template 2017) will be adapted to this review's study inclusion requirements and then tested on ten randomly selected studies (pilot test). One author will extract the data and another will verify it. Disagreements will be resolved in discussions between both authors. To organise the results, the studies were classified into categories established according to the major research topics that emerged from the content analysis.

\section{Subgroup analysis: NA.}

Sensitivity analysis: NA.

Language: None restriction.

Country(ies) involved: Portugal.

Keywords: Football; Soccer; Talent; Expert; Elite; Elite athlete; Talent identification; Talent development; Career transition; Career progression.

Dissemination plans: Publish the systematic review in a refereed journal and presenting it in a conference. 
Contributions of each author:

Author 1 - Vasco Cavaleiro.

Author 2 - Bruno Travassos.

Author 3 - Rui Mendes.

Author 4 - Hugo Sarmento. 